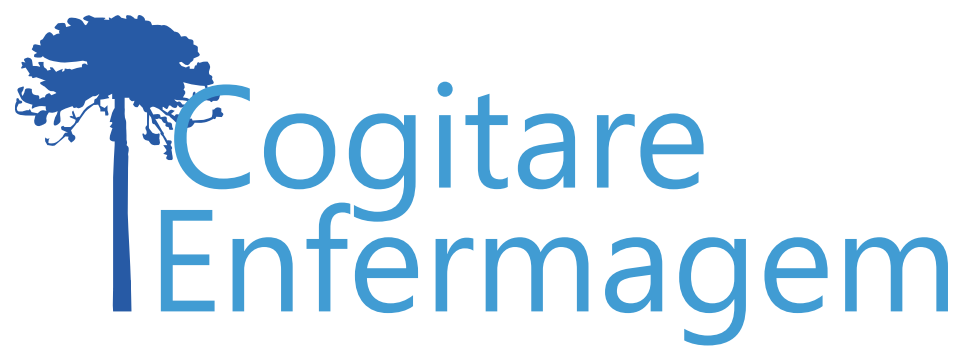

\title{
IMPLANTAÇÃO DE CATETER CENTRAL DE INSERÇÃO PERIFÉRI- CA POR ENFERMEIROS EM ADOLESCENTES
}

Nathália da Silva Pimentel Reis ${ }^{1}$, Maria Fabiane Galdino dos Santos², Dayana Carvalho Leite ${ }^{3}$, Helena Ferraz Gomes ${ }^{4}$, Ellen Marcia Peres ${ }^{5}$, Eugenio Fuentez Perez Junior ${ }^{6}$

\section{RESUMO}

Objetivo: analisar o perfil do processo de implantação do Cateter Central de Inserção Periférica, por enfermeiros capacitados, em adolescentes atendidos em um Hospital Universitário do Rio de Janeiro.

Método: estudo documental, descritivo, retrospectivo, de abordagem quantitativa, realizado entre janeiro de 2007 e dezembro de 2015. A amostra constituiu-se de 68 prontuários de adolescentes. Foram selecionados prontuários de adolescentes de 12 a 18 anos disponíveis no serviço de arquivo médico e que continham informações acerca do processo de implantação do cateter, e excluídos os dos pacientes que foram transferidos e não retiraram o cateter na instituição.

Resultados: constatou-se que 48 dos cateteres foram implantados em adolescentes do sexo masculino como diagnóstico médico doença onco-hematológica tendo como indicação terapêutica predominante a quimioterapia.

Conclusão: $O$ estudo contribuiu para a ampliação do conhecimento sobre o uso deste cateter, além de proporcionar subsídios para a realização de pesquisas e capacitação profissional.

DESCRITORES: Enfermagem; Adolescente; Cateterismo periférico; Cateterismo Venoso Central.

COMO REFERENCIAR ESTE ARTIGO: Reis NSP, Santos MFG, Leite DC, Gomes HF, Peres EM, Perez Junior EF. Implantação de cateter central de inserção periférica por enfermeiros em adolescentes. Cogitare enferm. [Internet]. 2019 [acesso em "colocar data de acesso, dia, mês abreviado e ano"]; 24. Disponível em: http://dx.doi. org/10.5380/ce.v24i0.55639.

Este obra está licenciado com uma Licença Creative Commons Atribuição 4.0 Internacional.

${ }^{1}$ Enfermeira. Especialista em Saúde do Adolescente. Hospital Universitário Pedro Ernesto. Rio de Janeiro, RJ. Brasil. (0) ${ }^{2}$ Enfermeira. Residente em Enfermagem em Pediatria do Instituto Fernandes Figueira. Rio de Janeiro, RJ. Brasil. 9 ${ }^{3}$ Enfermeira. Coordenadora de Enfermagem da Enfermaria do Núcleo de Estudos da Saúde do Adolescente do Hospital Universitário Pedro Ernesto. Rio de Janeiro RJ, Brasil. 1

${ }^{4}$ Enfermeira. Doutoranda em Enfermagem. Docente de Enfermagem da Universidade do Estado do Rio de Janeiro. Rio de Janeiro, RJ, Brasil. 1

${ }^{5}$ Enfermeira. Doutora em Saúde Coletiva. Docente de Enfermagem da Universidade do Estado do Rio de Janeiro. Rio de Janeiro, RJ, Brasil. 9

${ }^{6}$ Enfermeiro. Doutorando em Enfermagem. Docente de Enfermagem da Universidade do Estado do Rio de Janeiro. Rio de Janeiro, RJ, Brasil. 


\title{
PLACEMENT OF PERIPHERALLY INSERTED CENTRAL CATHETERS IN ADOLESCENTS BY NURSES
}

\begin{abstract}
Objective: To analyze the profile of peripherally inserted central catheters placement in adolescents admitted to a University Hospital of Rio de Janeiro by trained nurses.

Method: Documentary, descriptive, retrospective and quantitative study conducted between January 2007 and December 2015. The sample consisted of 68 medical records of adolescents. Medical records of adolescents aged 12-18 years available from the medical archive service and containing information about the process of placement of PICC catheters. The medical records of the patients transferred to other health centers and whose catheters were not removed in the institution were excluded.

Results: Of all the catheters inserted, 48 were placed in male adolescents diagnosed with onco-hematological disease, and chemotherapy was the predominant therapeutic indication. Conclusion: The present study contributed to improve the knowledge about the use of this type of catheter, which will be useful in future studies and in professional training.
\end{abstract}

DESCRIPTORS: Nursing; Adolescent; Peripheral catheterization; Central Venous Catheterization.

\section{IMPLANTACIÓN DE CATÉTER CENTRAL DE INSERCIÓN PERIFÉRICA POR ENFERMEROS EN ADOLESCENTES}

\author{
RESUMEN: \\ Objetivo: evaluar el perfil del proceso de implantación del Catéter Central de Inserción \\ Periférica, por enfermeros habilitados, en adolescentes atendidos en un Hospital Universitario \\ de Rio de Janeiro. \\ Método: estudio documental, descriptivo, retrospectivo, de abordaje cuantitativo, que se \\ realizó entre enero de 2007 y diciembre de 2015. La muestra se constituyó de 68 prontuarios \\ de adolescentes. Se seleccionaron prontuarios de adolescentes de 12 a 18 años disponibles \\ en el servicio de archivo médico, los cuales contenían informaciones acerca del proceso \\ de implantación del catéter; fueron excluidos los de los pacientes que se desplazaron y no \\ quitaron el catéter en el hospital. \\ Resultados: se constató que 48 de los catéteres fueron implantados en adolescentes del sexo \\ masculino como diagnóstico médico de enfermedad onco hematológica que tuvieron como \\ indicación terapéutica predominante la quimioterapia. \\ Conclusión: el estudio contribuyó para la ampliación del conocimiento sobre el uso del catéter, \\ además de proporcionar subsidios para la realización de investigaciones y perfeccionamiento \\ profesional.
}

DESCRIPTORES: Enfermería; Adolescente; Cateterismo periférico; Cateterismo Venoso Central. 
O Cateter Central de Inserção Periférica (Peripherally inserted central catheters-PICC) é um cateter venoso central de longa permanência, dentro da classificação dos cateteres semi-implantados; inserido em uma veia superficial da extremidade e que progride através de uma agulha e do fluxo de sangue, até o terço distal da veia cavasuperior ou inferior, adquirindo assim características de um cateter central, tendo em média um tempo máximo de permanência de dois anos e seis meses ${ }^{(1-3)}$.

Dentre os cateteres venosos centrais, o PICC apresenta uma melhor relação custobenefício, com menor custo, menores riscos, dispensa a utilização de sala cirúrgica, e pode ser inserido, inclusive, no leito de internação ou em residências; exige apenas um RX simples para confirmar sua localização após a inserção; apresenta eficiente resposta a tratamentos intravenosos com extremos de $\mathrm{pH}$ e osmolaridade ${ }^{(1-4)}$.

Os principais benefícios da utilização do cateter são a preservação do capital venoso periférico, a segurança medicamentosa na infusão de determinadas drogas, e apresentar uma menor exposição do paciente a dor. $O$ cateter possui também um menor risco de contaminação e maior tempo de permanência quando comparado a outros dispositivos intravasculares ${ }^{(5)}$.

Além dos benefícios relacionados ao custo-benefício, cabe ressaltar que o processo de inserção é um procedimento minimamente invasivo, a beira-leito que o enfermeiro pode realizar quando habilitado. No Brasil, a regulamentação técnica e legal para inserção e manuseio do PICC pelo enfermeiro é definida pelo Conselho Federal de Enfermagem (COFEN), através da Resolução n 258 de 2001, que em seu "art. $1^{\circ}$ torna lícito ao Enfermeiro a inserção do cateter central de inserção periférica (PICC)" e no "art. $2^{\circ}$, define que o enfermeiro para o desempenho de tal atividade, deverá ter qualificação e/ou capacitação profissional"(6).

Desde a promulgação da Resolução supracitada, o enfermeiro vem assumindo com desenvoltura esta atribuição na prática clínica. Vale destacar que muitos avanços ocorreram nos cuidados relacionados à prática de inserção do PICC com a produção de conhecimentos científicos na área da enfermagem, que tem contribuído para qualificação e para uma prática clínica cada vez mais segura e eficaz ${ }^{(7)}$.

Estudo coreano sobre a atuação de enfermeiras especialistas em PICC constatou que as terapias de infusão que levaram as enfermeiras a inserirem o PICC foram eficazes e seguras, com taxas de complicação relativamente baixas, demonstrando o importante papel desempenhado no aumento do uso deste tipo de cateter ${ }^{(8)}$.

Ainda, no que se refere ao conhecimento sobre a indicação do uso do cateter, estudos ratificam a importância do uso de PICC em pacientes oncológicos, que representa a mitigação do sofrimento físico daqueles que apresentam vulnerabilidades diversas frente à hospitalização e ao processo de adoecimento. Desta forma, o PICC constitui uma opção segura aos pacientes em terapia intravenosa $(\mathrm{TI})$ contribuindo para a melhoria da qualidade de vida ${ }^{(7-9)}$.

No entanto, apesar dos estudos na área de neonatos, crianças e adultos internados em terapia intensiva apresentarem a utilização do PICC como uma prática rotineira(10-12), são incipientes os estudos envolvendo adolescentes hospitalizados em uso deste cateter.

Assim, o presente estudo tem por objetivo analisar o perfil do processo de implantação do PICC, por enfermeiros capacitados, em adolescentes atendidos em um Hospital Universitário do Rio de Janeiro, Brasil, visando a colocar à disposição dos interessados a experiência desenvolvida nesse serviço que integra a rede de atenção em saúde.

Trata-se de um estudo desenvolvido em uma enfermaria de assistência integral à saúde de adolescentes, cujo objeto é o perfil do processo de implantação do Cateter Central de Inserção Periférica (PICC). 
Estudo descritivo, retrospectivo e documental, de abordagem quantitativa, sobre o processo de implantação do Cateter Central de Inserção Periférica (PICC), no período entre janeiro de 2007 e dezembro de 2015. Realizado em uma enfermaria especializada em saúde do adolescente de um Hospital Universitário do Estado do Rio de Janeiro, composta por 16 leitos, sendo oito para o sexo feminino e oito para o sexo masculino, e que responde pela atenção integral desse público com idade compreendida entre 12 e 18 anos.

O cenário de estudo é um centro de referência para internação de adolescentes de ambos os sexos com quadros clínicos e cirúrgicos que requeiram investigação diagnóstica e tratamento, com exceção de adolescentes em trabalho de parto ou em surto psicótico.

A assistência neste centro é prestada por uma equipe multiprofissional, constituída por profissionais da Enfermagem, da Medicina, da Nutrição, do Serviço Social, da Psicologia e da Fisioterapia. Contudo, o Enfermeiro, segundo a Resolução do COFEN n ${ }^{\circ} 258 / 2001$, é guem realiza a implantação e a manutenção do PICC nos pacientes com indicação clínica. É importante destacar que a decisão de implantação do PICC é uma ação conjunta com a equipe médica e o procedimento segue as normas e protocolos já estabelecidos pela instituição(6).

Os adolescentes a serem submetidos à implantação do PICC são aconselhados e orientados quanto aos cuidados de manutenção, sendo acompanhados pelo enfermeiro ou residente de enfermagem. As trocas de curativos e outros procedimentos são efetuados a cada sete dias ou sempre que houver necessidade.

A amostra deste estudo constitui-se de 68 prontuários de adolescentes que foram submetidos ao procedimento de implantação do cateter central de inserção periférica, e acompanhados no serviço desde a inserção até a retirada do cateter.

Foram utilizados os seguintes critérios inclusão: prontuários de adolescentes de 12 a 18 anos disponíveis no serviço de arquivo médico e que continham informações acerca do processo de implantação do PICC. Foram excluídos prontuários de pacientes que foram transferidos e não retiraram o cateter na instituição.

A coleta de dados ocorreu nos meses de julho a setembro de 2016 por meio da análise de prontuários e impressos próprios do setor, referentes à implantação e manutenção do PICC em adolescentes.

O impresso utilizado para esse procedimento é preenchido, assinado e carimbado pelo enfermeiro responsável pela inserção e/ou retirada do PICC. Para fins deste estudo, as variáveis avaliadas foram: sexo, idade e diagnóstico médico, conforme Classificação Internacional de Doenças-CID 10, indicação clínica, local de punção, tempo de permanência do cateter e o motivo de retirada.

Os dados após coletados foram digitados e tabulados eletronicamente e, sequencialmente, realizou-se a consolidação no programa Microsoft Excel, com análise estatística descritiva simples, e de distribuição de frequências dos dados que representaram as variáveis do estudo. Os resultados foram discutidos à luz da literatura científica disponível.

Para atender aos critérios éticos, a pesquisa foi submetida ao Comitê de Ética em Pesquisa da instituição e aprovado sob o parecer de $n^{\circ} 1.645 .428$. Por se tratar de uma pesquisa retrospectiva de análise documental do prontuário e impressos próprios do setor, dispensa a assinatura do Termo de Consentimento Livre e Esclarecido. 
$48(71, \%)$ eram do sexo masculino. Quanto à faixa etária, a idade mínima e máxima eram respectivamente, 12 e 18 anos, sendo a média de 14,6 anos, com um desvio-padrão de 2,7 anos.

Ao mapear o diagnóstico médico dos pacientes submetidos ao procedimento, constatou-se um predomínio das doenças onco-hematológicas, com destaque para as leucemias e linfomas representados por 31 (46\%) casos, como apresentado na Tabela 1.

Tabela 1 - Diagnósticos médicos (CID10) de adolescentes hospitalizados em uso de PICC $(\mathrm{N}=67)^{\star}$, Rio de Janeiro, RJ, Brasil, 2007-2015

\begin{tabular}{llc} 
Variáveis & $\mathbf{n}$ & $\%$ \\
\hline Leucemias & 17 & 25 \\
\hline Linfoma & 14 & 21 \\
\hline Osteomielite & 12 & 18 \\
\hline Artrite séptica & 1 & 1,5 \\
\hline Derivação ventricular externa + infecção fúngica & 2 & 3 \\
\hline Fibrose cística & 3 & 4,5 \\
\hline Mielomeningocele + bexiga neurogênica & 1 & 1,5 \\
\hline Endocardite + sepse & 1 & 1,5 \\
\hline Vírus da imunodeficiência adquirida humana + meningite criptocócica & 1 & 1,5 \\
\hline Paralisia facial aguda + lesão sacral infectada & 1 & 1,5 \\
\hline Vírus da Imunodeficiência adquirida + infecção pulmonar & 1 & 1,5 \\
\hline Linfoadenomegalia a esclarecer & 3 & 4,5 \\
\hline Actinomicose & 1 & 1,5 \\
\hline Neurosífilis congênita & 2 & 3 \\
\hline Aplasia de medula óssea grave + amigdalite & 1 & 1,5 \\
\hline Paracoccidioidomicose disseminada & 2 & 3 \\
\hline Tumor não específico & 2 & 0,3 \\
\hline Hepatomegalia e esplenomegalia & 1 & 1,5 \\
\hline Puerpério tardio + Síndrome de Dress & 1,5 \\
\hline TOTAL & 100
\end{tabular}

Fonte: Dados do Estudo

*Não houve registro de um (1) paciente quanto ao diagnóstico médico.

Dentre as indicações de implantação do cateter, destaca-se a administração de quimioterapia isolada em 28 (41\%) ou associada à antibioticoterapia ou anfotericina B três (4\%), o que demonstra que a quimioterapia foi a finalidade em $31(45 \%)$ casos. As demais indicações encontradas foram: a antibioticoterapia em 27 (39\%), administração de anfotericina B em cinco (7\%), infusão de timoglobulina em um (1\%), e quatro (5\%) dos cateteres não possuíam registro.

Em relação ao acesso venoso de escolha, as veias predominantemente puncionadas foram as dos membros superiores (MMSS), cefálica e basílica, em 50 (73\%) das implantações 
do PICC, enquanto a veia jugular foi a escolha em 14 (21\%). Já o restante dos cateteres que corresponde a quatro $(6 \%)$ não possuía registro quanto à veia de punção.

Ao avaliar a variável tempo de permanência, dos 68 cateteres implantados, destacase a ausência de registro da data de retirada em 41 (60\%). Verificou-se, também, que apenas um cateter apresentava tempo de uso igual a zero dia, o mesmo não progrediu sendo indicado sua retirada. Os demais 26 cateteres implantados estão descritos no Gráfico 1:

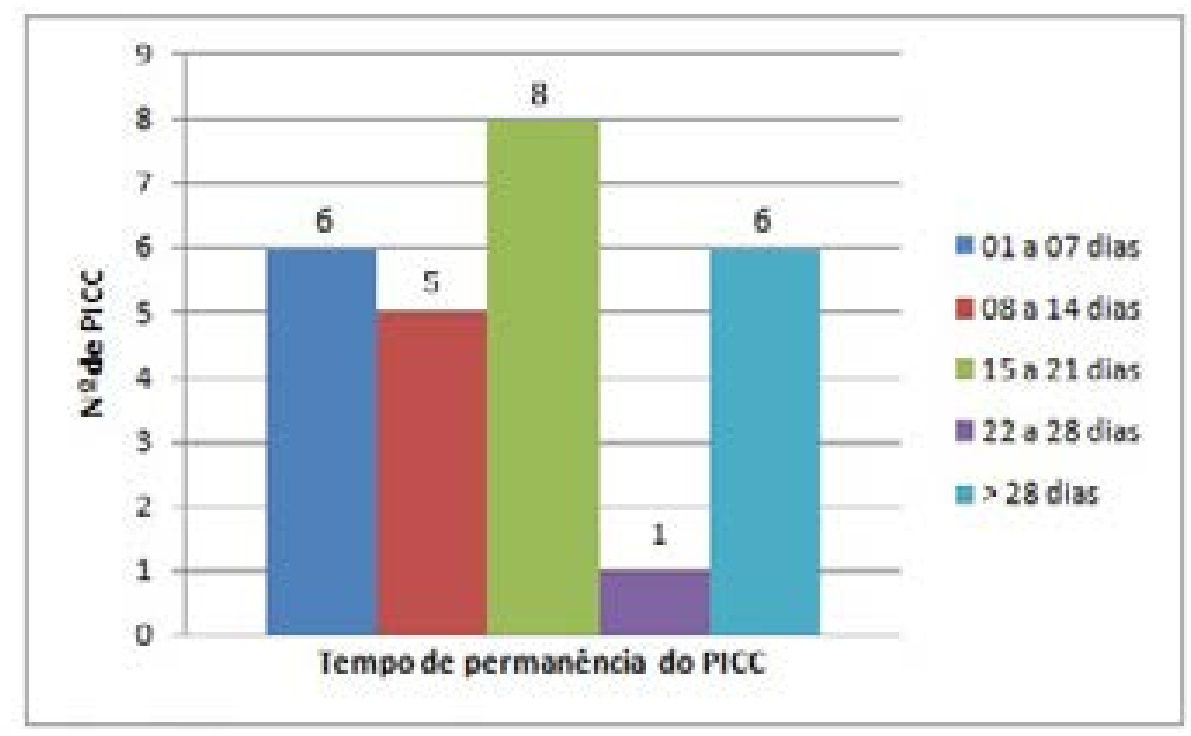

Gráfico 1 - Tempo de permanência em dias dos PICC implantados em adolescentes hospitalizados que foram registrados ( $\mathrm{N}=26)$, Rio de Janeiro, RJ, Brasil, 2007-2015

$\mathrm{Na}$ avaliação da variável motivo de retirada do cateter, prevaleceu a ausência de registro do motivo de retirada em 36 (53\%) dos casos. No entanto, os demais motivos de retirada, que correspondem a 32 situações (47\%), predominaram os casos de término da terapêutica e obstrução do cateter, ambos com oito (25\%), seguidos dos problemas relacionados ao cateter (fratura do cateter) com sete (22\%), sendo o deslocamento do mesmo e ocorrência de complicações infecciosas com quatro (12,5\%) cada, e apenas um (3\%) cateter relativo ao não progresso.

\section{DISCUSSÃO}

As doenças oncológicas principalmente as de origem hematológicas figuraram como os principais diagnósticos dos adolescentes que utilizaram o PICC no período analisado. Tal condição pode estar relacionada ao fato de que estas patologias apresentam alta incidência e prevalência em adolescentes no Brasil, sendo as neoplasias malignas do tecido linfático e hematopoiético as que representam cerca de $50 \%$ das mortes por câncer na faixa etária de dez a 14 anos, e $40 \%$ do grupo de 15 a 19 anos $^{(13)}$.

Neste sentido, os dados recentes do Instituto Nacional de Câncer José Gomes Alencar da Silva (INCA) ${ }^{(10)}$ ratificam que o percentual mediano de neoplasias é de $3 \%$ na população de crianças e adolescentes (de 0 a 19 anos), sendo observada a maior frequência de leucemias nos grupos de 0 a 14 anos: 33,2\%; de 0 a 19 anos: 25,6\%. Os linfomas têm frequência de $13,6 \%$ na faixa etária de 0 a 19 anos, enquanto a mediana das taxas médias de incidência, nesta mesma faixa etária, foi de 139,99 por milhão, apresentando pico etário em adolescentes de 15 a 19 anos ${ }^{(14-15)}$. 
Para o tratamento das doenças oncológicas, o estabelecimento de um acesso venoso de longa permanência é fundamental, pois gera confiança durante a terapia infusional de drogas vesicantes e irritantes. Destaca-se que as principais indicações do PICC no cenário pesquisado foram para administração de quimioterapia antineoplásica ${ }^{(16)}$.

No que diz respeito à indicação para utilização do PICC como via segura à administração de soluções parenterais, identificou-se que face ao diagnóstico doença onco-hematológica cujo tratamento é realizado através de infusões de quimioterápicos por tempo prolongado, a utilização do cateter é indicada devido ao uso de drogas irritantes e vesicantes, o que corrobora os dados encontrados ${ }^{(17)}$.

É importante destacar que o PICC é considerado um acesso venoso eficaz, ao constituir uma via segura para administração de antibióticos, nutrição parenteral total e quimioterápicos, além de apresentar baixo índice de infecção(5,18), permitindo, portanto, a prevenção de eventos adversos decorrentes da infusão e a segurança do paciente.

Os resultados do estudo apontaram que a administração de quimioterapia isolada ou associada à antibioticoterapia ou anfotericina B foram as principais indicações para implantação do PICC. Diametralmente oposto a estes resultados, uma pesquisa desenvolvida em neonatologia revelou que as principais drogas administradas através do cateter de inserção periférica são a Nutrição Parenteral Total (NPT) com administração simultânea e contínua de heparina ${ }^{(19)}$.

Dentre as complicações mais comuns decorrentes do uso do PICC em pacientes oncológicos estão as alergias de pele, seguida da oclusão do cateter e da retirada acidental, e em menor incidência, a trombose venosa de membro superior e infecção de corrente sanguínea(20).

Este estudo evidenciou predomínio de término da terapêutica e obstrução do cateter, seguidos dos problemas relacionados ao mesmo (fratura do cateter), deslocamento e ocorrência de complicações infecciosas.

Outro aspecto a ser ressaltado refere-se ao local de inserção do PICC. Estudo realizado aponta que as condições ideais para inserção do cateter são: observar o estado da veia a ser puncionada, se é palpável, calibrosa, apresentando menor curvatura, bem como integridade da pele no local de escolha, sem hematomas, edemas, sinais de infecção ou alteraçōes anatômicas ${ }^{(21)}$.

Autores ${ }^{(10,21)}$ referem que as veias mais indicadas são a basílica e a cefálica, pois apresentam menor número de válvulas, são de maior calibre, têm anatomia favorável, e assim, facilitam a realização e a troca de curativos, além de terem menor trajeto até a veia cava superior, motivos pelos quais verificou-se na amostra uma alta taxa de inserção do cateter por essas veias.

Ao analisar o tempo de permanência, constatou-se que a maioria dos cateteres permaneceram por um período superior a 15 dias. Estudos brasileiros descrevem a permanência do PICC em crianças num período de 11 a 14 dias ${ }^{(10,22)}$. Compreende-se, deste modo, que os dados encontrados apresentam-se consonantes com a realidade nacional em perspectiva positiva de análise.

No contexto asiático, a experiência de inserção de PICC em pacientes com doenças hematológicas foi descrito num estudo que analisou a segurança da colocação destes cateteres, evidenciando que a duração global da cateterização foi de 8.089 dias, com duração média de 57 dias, e que a quimioterapia foi administrada por meio de 107 cateteres dos 142 inseridos ${ }^{(16)}$.

Dentre os motivos de retirada não programada do cateter, ou seja, antes do término da terapia farmacológica, verificou-se: a obstrução, problemas como fratura do cateter, desposicionamento, infecção e não progressão do mesmo. No que diz respeito à obstrução, estudos apontam que as principais causas estão relacionadas à ausência da lavagem dos 
cateteres com solução salina a 0,9\% recomendada como forma de garantir a permeabilidade do cateter e prevenir a formação de coágulos e de fibrina, ou a precipitação entre fármacos incompatíveis, enfatizando a necessidade de treinamento da equipe de enfermagem ${ }^{(23-24)}$.

Somam-se ainda a essas causas mencionadas para retiradas não programadas dos cateteres a posição incorreta de sua ponta. Estudos apontam para a necessidade da correta confirmação do posicionamento do cateter após sua passagem e a realização de fixação e avaliaçõ̃es diárias a fim de verificar a manutenção correta da posição e ou da sua exteriorização ${ }^{(10,22-25)}$.

No que diz respeito à infecção da corrente sanguínea, estudos referem que, quanto maior o tempo de permanência, maior é o risco de infecção, e apontam como atividades essenciais para a sua prevenção a realização de curativos com filme transparente, a adoção de medidas de assepsia e antissepsia, e o treinamento constante da equipe que manipula o PICC (10,22-24).

A não progressão do cateter ou a dificuldade de sua inserção ainda é referida em estudos como uma ocorrência possível na introdução do PICC, atribuindo a isso as seguintes causas: sangramento excessivo, obstrução por válvulas venosas, anatomia venosa aberrante, resistência no trajeto causado por venoespasmo, esclerose, posição incorreta do cateter ou do paciente, bifurcação venosa ou dissecção de veia prévia(21-26).

Dentre as limitações do estudo, destaca-se a falha de preenchimentos de algumas informações nos documentos do serviço, ocasionando um prejuízo na precisão dos dados, principalmente nas variáveis tempo de permanência e motivo de retirada do PICC.

\section{CONCLUSÃO}

Ao analisar o perfil do processo de implantação do PICC em adolescentes a partir dos dados disponíveis nos prontuários, no período de janeiro de 2007 a dezembro de 2015, o estudo evidenciou que o perfil clínico dos adolescentes submetidos à inserção do PICC foram os acometidos pelas doenças oncológicas, principalmente as de origem hematológica.

A principal indicação da implantação do PICC foi a necessidade de uma via endovenosa segura e de longa permanência para administração de drogas irritantes e vesicantes.

Os resultados deste estudo sugerem que o PICC na população estudada se mostrou seguro para administração de quimioterapia, pois a média acima de 15 dias de sua utilização já o caracteriza como um cateter de longa duração, possibilitando seu uso até o término do tratamento.

Embora as vantagens destes cateteres sejam indubitáveis, foram evidenciadas complicações, tais como obstrução, problemas como fratura do dispositivo, seu desposicionamento, infecção e não progressão que culminaram na sua retirada.

Espera-se que o resultado desse estudo venha contribuir para estimular e encorajar enfermeiros de serviços públicos e privados, a investirem em sua qualificação profissional para se habilitarem na técnica de inserção, manuseio e manutenção de Cateter Central de Inserção Periférica (Peripherally inserted central catheters-PICC) e, assim, oferecer aos usuários assistência de qualidade e livre de riscos. Não menos importante, embora de relevância secundária, mas que merece ser assinalada é a contribuição do uso do PICC na redução de gastos de outros materiais e insumos no contexto da assistência. 
1. Ministério da Saúde (BR). Instituto Nacional de Câncer José Gomes Alencar da Silva. Ações de enfermagem para o controle do câncer: uma proposta de integração ensino-serviço. [Internet].Rio de Janeiro: INCA; 2008. [acesso em 11 fev 2017] Disponível em: http://bvsms.saude.gov.br/bvs/publicacoes/ acoes enfermagem controle cancer.pdf.

2. Phillips LD. Manual de terapia intravenosa. 2. ed. Porto Alegre (RS): Artmed; 2001.

3. Jakitsch CB, Carvalho DPL, Posso MBS, Machado RC, Giaretta VMA. Cateter central de inserção periférica: utilização no vale do paraíba paulista. Rev. pesqui. cuid. fundam. (Online).[Internet]. 2016 [acesso em 11 fev 2017];8(2). Disponível em: http://dx.doi.org/10.9789/2175-5361.2016.v8i2.4280-4289.

4. Rodrigues ZS, Chaves EMC, Cardoso MVL. Atuação do enfermeiro no cuidado com o cateter central de inserção periférica no recém-nascido. Rev. bras. enferm.[Internet]. 2006 [acesso em 10 dez 2016];59(5) Disponível em: http://dx.doi.org/10.1590/S0034-71672006000500006.

5. Di Santo MK, Takemoto D, Nascimento RG, Nascimento AM, Siqueira E, Duarte CT, et al. Cateteres venosos centrais de inserção periférica: alternativa ou primeira escolha em acesso vascular? J. vasc. bras.[Internet]. 2017[acesso em 23maio 2018];16(2). Disponível em: http://dx.doi.org/10.1590/1677$\underline{5449.011516 .}$

6. Conselho Federal de Enfermagem. Resolução n. 258, de 12 de julho de 2001. Inserção de Cateter Central Periférico, pelos enfermeiros. Rio de Janeiro: COFEN: 2001.

7. Avelar AFM, Peterlini MAS, Pedreira MLG. Assertividade e tempo de permanência de cateteres intravenosos periféricos com inserção guiada por ultrassonografia em crianças e adolescentes. Rev. Esc. Enferm. USP.[Internet].2013 [acesso em 13 fev 2017];47(3). Disponível em: http://dx.doi.org/10.1590/ S0080-623420130000300003.

8. Park JY, Kim HL. A Comprehensive Review of Clinical Nurse Specialist-Led Peripherally Inserted Central Catheter Placement in Korea: 4101 Cases in a Tertiary Hospital. J InfusNurs[Internet]. 2015 [acessoem 10 fev 2017];38(2). Disponível em: http://www.nursingcenter.com/pdfjournal?AID=2760835\&an=00129804201503000-00005\&Journal ID=237151\&Issue ID=2760744.

9. Bergami CMC, Monjardim MAC, Macedo CR. Utilização do cateter venoso central de inserção periférica (PICC) em oncologia pediátrica. REME - Rev Min Enferm[Internet] 2012;16(4) [acesso em 10 fev 2017]. Disponível em: http://www.reme.org.br/artigo/detalhes/559.

10. Baggio MA, Bazzi FCS, Bilibio CAC. Cateter central de inserção periférica: descrição da utilização em UTI Neonatal e Pediátrica. Rev. GaúchaEnferm. [Internet]. 2010 [acesso em 10 dez 2016];1(1). Disponível em: http://dx.doi.org/10.1590/S1983-14472010000100010.

11. Lamblet LCR, Guastelli LR, Júnior Moura DF, Alves MAY, Bittencourt AC, Teixeira APP, et al. Cateter Central de inserção Periférica em terapia intensiva de Adultos. Rev. bras. ter. intensiva. [Internet]. 2005 [acesso em 10 dez 2016];17(1). Disponível em:http://files.enfermeiros-intensivistas.webnode. pt/200000066-75ee276e95/CLIQUE\%20AQUI\%20PICC\%20em\%20Adultos.pdf.

12. Jantsch LB, Neves ET, Arrué AM, Kegler JJ, Oliveira CR. Utilização do cateter central de inserção periférica em neonatologia. Rev. baiana enferm.[Internet]. 2014 [acesso em 10 dez 2016];28(3). Disponível em: https://portalseer.ufba.br/index.php/enfermagem/article/view/10109.

13. Presti PF, Macedo CRD, Caran EM, Rodrigues AHD, Petrilli AS. Estudo epidemiológico de câncer na adolescência em centro de referência. Rev. paul. pediatr.[Internet]. 2012 [acesso em 12 jan 2017];30(2). Disponível em: http://dx.doi.org/10.1590/S0103-05822012000200009.

14. Instituto Nacional de Câncer (BR). Incidência, mortalidade e morbidade hospitalar por câncer em crianças, adolescentes e adultos jovens no Brasil: informações dos registros de câncer e do sistema de mortalidade. [Internet].Rio de Janeiro: INCA; 2016. [acesso em 11 fev 2017]. Disponível em: http:// www1.inca.gov.br/wcm/incidencia/2017/pdf/versao-completa.pdf.

15. Bleyer WA. Cancer in older adolescents and young adults: epidemiology, diagnosis, treatment, survival, and importance of clinical trials. MedPediatrOncol[Internet]. 2002 [acesso em 20 fev 2017]; 38(1). 
Disponível em: http://onlinelibrary.wiley.com/doi/10.1002/mpo.1257/epdf?r3 referer=wol\&tracking action=preview click\&show checkout=1\&purchase referrer=www.google.com.br\&purchase site license=LICENSE DENIED.

16. Hashimoto Y, Fukuta T, Maruyama J, Omura H, Tanaka T. Experience of Peripherally Inserted Central Venous Catheter in Patients with Hematologic Diseases. Intern. Med. [Internet]. 2017 [acesso em 10 mar 2017];56(4). Disponível em: http://dx.doi.org/10.2169/internalmedicine.56.7625.

17. Machado LBL, Moura DA, Cunha LBC, Cunha KCS. Característica dos cateteres e de crianças portadoras de doença oncohematológica. Cogitareenferm. [Internet]. 2017 [acesso em 20 set 2017];22(1). Disponível em: http://dx.doi.org/10.5380/ce.v22i1.48448.

18. Franceschi AT, Cunha MLC. Eventos adversos relacionados ao uso de cateteres venosos centrais em recém-nascidos hospitalizados.Rev. Latino-Am. Enfermagem. [Internet]. 2010 [acesso em 23mai 2018];18(2). Disponível em: http://dx.doi.org/10.1590/S0104-11692010000200009.

19. Giraldo-Montoya DI, Quirós-Jaramillo A, Mejía-Cadavid LAM. Manejo de catéteres centrales de inserción periférica en reciénnacidos. Aquichán. [Internet]. 2008 [acesso em 10 mar 2017];8(2). Disponível em: http://www.scielo.org.co/scielo.php?script=sci arttext\&pid=S1657-59972008000200012\&lng=en\&nr $\underline{m}=$ iso.

20. Kang J, Chen W, Sun W, Ge R, Li H, Ma E, et al. Peripherally inserted central catheter-related complications in cancer patients: a prospective study of over 50,000 catheter days. J Vasc Access. [Internet]. 2017 [acesso em 20 ago 2017];18(2). Disponível em: http://dx.doi.org/10.5301/jva.5000670.

21. Martins C, Oselame GB, Neves EB. Cateter central de inserção periférica: revisão sistemática. Rev. Aten. Saúde. [Internet]. 2016 [acesso em 20 abril 2017];14(47). Disponível em: http://dx.doi.org/10.13037/ ras.vol14n47.3358.

22. Costa $P$, Kimura AF, Vizzotto MPS, Castro TE, West A, Dorea E. Prevalência e motivos de remoção não eletiva do cateter central de inserção periférica em neonatos. Rev. Gaúcha Enferm. [Internet]. 2012 [acesso em 10 ago 2017];33(3). Disponível em: http://dx.doi.org/10.1590/S1983-14472012000300017.

23. Costa P, Paiva ED, Kimura AF, Castro TE. Fatores de risco para infecção de corrente sanguínea associada ao cateter central de inserção periférica em neonatos. Acta paul. enferm. [Internet]. 2016[acesso em 10 ago 2017];29(2). Disponível em: http://dx.doi.org/10.1590/1982-0194201600023.

24. Silva RMM, Lui AM, Ferreira H, Franca AFO, Lala ERP, VieraCS.Analysingthe use of peripherally inserted central catheter in neonatology. Rev. enferm. UFPE on line. [Internet]. 2016 [acesso em 10 ago 2017];10(Suppl 2). Disponível em: https://periodicos.ufpe.br/revistas/revistaenfermagem/article/ view/11022.

25. Oliveira CR, Neve ET, Rodrigues EC, Zamberlan KC, Silveira A. Cateter central de inserção periférica em pediatria e neonatologia: possibilidades de sistematização em hospital universitário. Esc. Anna Nery [Internet]. 2014 [acesso em 10 fev 2017];18(3). Disponível em: http://dx.doi.org/10.5935/1414$\underline{8145.20140054 .}$

26. Sharpe E, Pettit J, Ellsbury DL. A national survey of neonatal peripherally inserted central catheter (PICC) practices. AdvNeonatal Care. [Internet]. 2013 [acesso em 10 ago 2017];13(1). Disponível em: http://dx.doi.org/10.1097/ANC.0b013e318278b907.

Recebido: 09/10/2017

Finalizado: 17/12/2018

Autor Correspondente:

Nathália da Silva Pimentel Reis

Universidade do Estado do Rio de Janeiro

R. Parima, 114 - 21250-430 -Rio de Janeiro, RJ, Brasil

E-mail: nathaliapimentelenf@gmail.com 
Contribuição dos autores:

Contribuições substanciais para a concepção ou desenho do estudo; ou a aquisição, análise ou interpretação de dados do estudo - NSPR, MFGS, DCL, HFG, EMP, EFPJ

Elaboração e revisão crítica do conteúdo intelectual do estudo - NSPR, MFGS, DCL, HFG, EMP, EFPJ

Aprovação da versão final do estudo a ser publicado - NSPR, MFGS, DCL, HFG, EMP, EFPJ

Responsável por todos os aspectos do estudo, assegurando as questões de precisão ou integridade de qualquer parte do estudo - NSPR, MFGS, DCL, HFG, EMP, EFPJ 